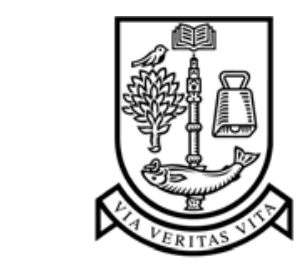

UNIVERSITY

of

GLASGOW

Purchase, H.C. (2006) Student compliance with ethical guidelines: the Glasgow ethics code. Innovations in teaching and learning in information and computer sciences: ITALICS 5(2).

http://eprints.gla.ac.uk/3681/ 


\title{
Student compliance with ethical guidelines: The Glasgow Ethics Code
}

\author{
Helen C Purchase \\ Department of Computing Science \\ University of Glasgow \\ hcp@dcs.gla.ac.uk \\ www.dcs.gla.ac.uk/ hcp/ethics
}

\begin{abstract}
While disciplines like medicine and psychology have for several years followed strict procedures for ethical approval of experiments involving humans, only recently has the use of human participants within Computing Science been subject to the same scrutiny. Although we may wish to put a case forward for Computing Science to be exempt from such formal ethics procedures, funding bodies and universities typically insist that we seek the same approval as other disciplines for our experiments, including any use of human participants by our students during the course of their studies. We have introduced a simple, scalable ethics procedure for student assessment, that identifies ethical concerns, yet does not overwhelm the limited staff resources available for supporting this initiative. The process is based around a form of triage that filters the approximately 8000 assessments that are submitted annually. This paper summarises this procedure, discusses the underlying assumptions, and outlines the problems encountered.
\end{abstract}

\section{Keywords}

Ethical approval, human participation, student assessment.

\section{INTRODUCTION}

In 2002, following a review of the existing procedures for conducting research, the Senate at the University of Glasgow decided that a more consistent approach should be adopted throughout the university. This initiative was partly instigated by a statement from the Wellcome Trust, which provides substantial research funding to our university, that all funding would be withdrawn unless they were satisfied that ethical procedures

\footnotetext{
Permission to make digital or hard copies of all or part of this work for personal or classroom use is granted without fee provided that copies are not made or distributed for profit or commercial advantage and that copies bear this notice and the full citation on the first page. To copy otherwise, to republish, to post on servers or to redistribute to lists, requires prior specific permission.
}

(c) 2005 HE Academy for Information and Computer Sciences were being followed appropriately throughout the whole university. From October 2002 the University required that all research involving human participants or human data or material be subject to formal ethical review. This not only affects disciplines such as medicine but also covers the evaluation of software involving potential end-users, including other students.

The university devolved responsibility for monitoring ethical procedures to the faculties. Our faculty of Information and Mathematical Sciences includes the department of Psychology which has always had a department ethics committee. This is based around the British Psychology Society guidelines (BPS, 2004) and monitors both student projects and research projects conducted by academic or research staff. The introduction of the Faculty Ethics Committee meant a shift in decision making: all student projects and assessments using human participants would be dealt with by the respective department ethics committees, while all human data collection performed by academic or research members of staff, or postgraduate research students require prior approval from the Faculty Ethics Committee through the submission of a form. This form, while asking specific questions regarding the ethical treatment of participants, also requires a clear statement of the purposes of the research, and a detailed description of the experiment (including hypotheses, conditions etc.) In Psychology, even final year students are required to complete this form for their projects, as it is considered part of their professional training.

The Computing Science Department Ethics Committee was therefore charged with the responsibility of monitoring the use of human participants for all undergraduate and taught postgraduate assessments or projects. We were told that we needed to put procedures into place to ensure that ethics guidelines are being followed, to have these procedures approved by the Faculty Ethics Committee, and to be prepared to be audited at any time. 


\section{The Problems for COMPUTING SCIENCE}

There are clearly two different uses of human participation within computing science: formal experiments that collect data in order to test hypotheses, and both formative and summative evaluation of software as part of the software engineering life cycle. While we would have liked this latter use of human participation to be made exempt from the ethics requirements, we were informed that no special case could be made for the Computing Science Department, and all human participation was required to clearly conform to ethical procedures.

Investigation of some of our current modules revealed several examples of students being required to use human participants as part of their assessment. In our first year, students develop a paper-based prototype of a mobile device for accessing timetable and booking information about flights. They choose an evaluation technique, design an evaluation plan, and evaluate their design, typically with other students in the class during a scheduled lab session. The design is improved iteratively in response to the results of the evaluations. Students submit a report describing their evaluation method, their results, and design improvements suggested by the evaluation.

Our final year and taught postgraduate projects mostly entail the design and implementation of a software system. Although a few projects are research-based and use controlled experiments, evaluation activities performed within these projects are typically of a formative nature, as an essential part of the iterative design cycle. The formative evaluations typically take the form of interviews or questionnaires. Participants may not be current students and, depending on the nature of the system, students may wish to evaluate the systems use with participants under the age of 16 (typically family members). The report submitted by students normally includes a description of their evaluation method, their results, the use of formative evaluations during the design, and design improvements suggested by a final evaluation. Some of these projects involve close cooperation with end-users ranging from the local police through to art galleries. In the past, some projects have focused on the design of software for schools. Others have developed programs to help in the assessment of psychological disorders. Topics like these raise obvious ethical issues; however, the majority of projects do not, and have only trivial ethical concerns.

One of the most difficult issues to overcome was introducing these ethical procedures to members of a department that had never had to consider them before and who, in the main, considered them wholly unnecessary. Many academics complained that these procedures were being introduced when there is no actual problem to be addressed, about the increased bureaucracy, and the fact that in our discipline risks are slight. Comments from academic members of staff included:

- Are we aware of any instances where colleagues' students adopted practises that were ethically dubious, such as coercing reluctant individuals into evaluation their web interfaces etc.? Did the university really intend that such heavy procedures should be applied in our area?

- Everyone uses a computer these days what risks can there be?

- What is the penalty for us, as academics, if we do not ensure that these guidelines are being followed?

- What is penalty for students who do not follow the ethical guidelines?

- Can the whole department put forward a case for not having to follow the ethical guidelines, because of the nature of our work?

\section{OUR Ethics Procedures}

We were advised by the Faculty Ethics Committee to be conservative in our approach, and to devise a method that was our interpretation of the minimum requirements according to the formal guidelines. We also wished to make it clear that we considered following these ethical procedures as part of the 'ethical education' of our students, to be introduced in their first year of study.

The ethical standard that we are obliged to conform to is that of the British Psychological Society [1]. This offers clear benefits in that it is a recognised external source and has already been widely used within the University. However, this document is somewhat verbose and not well suited to the particular needs of a computing department. We distilled these guidelines into 12 clear ethical points, thus making them easier to be explained to students and providing a clear list against which the ethics of an evaluation or experiment can be judged.

Our first attempt entailed a clumsy system of dual form-filling, and placed the responsibility clearly with the module co-ordinator or project supervisor. After getting feedback from academic members of staff, our Head of Department, and the Faculty Ethics Committee, a lighter-weight system was devised.

The procedure is based on the assumption that all assessments and projects will conform to the 12point check list, and requires that the student sign and submit a form to state that this is the case. It then makes provision for formal Department Ethics Committee approval to be given to any evaluations 
conducted as part of projects whose procedures fall outwith these guidelines. In these rare cases, the student is required to submit an ethics approval request form the Department Ethics Committee, a form modelled on the ethics form required by the Faculty Ethics Committee for research projects.

\subsection{The Glasgow Ethics Code check-list}

The minimal resources that the students require are the 12-point check list form ('The Glasgow Ethics Code') that they need to sign, as well as example introduction and debriefing scripts. Students whose evaluation does not comply with any one of the 12 points and who have applied for formal approval from the Department Ethics Committee also require an example consent form and a take-away information sheet. These, together with instructions for use, are provided on an easily accessible website.

The 12 statements on the form that the students sign are shown in bold below, interspersed with comments:

\section{Participants were not exposed to any risks greater than those encountered in their normal working life.}

This will usually be the case for software evaluation: the counter example that is often given is the evaluation of navigation software running on PDA while crossing a busy road!

2. The experimental materials were paperbased, or comprised software running on standard hardware.

Currently, our definition of 'standard hardware' is a laptop or desktop PC, which will cover the majority of student projects. Projects using any other hardware (eg PDAs, tactile or audio equipment) need to apply for approval - in assessing these applications we typically ensure that equipment has been recently safety tested, and that the evaluation procedure allows for sufficient training and is not too physically or cognitively demanding for participants who may be unfamiliar with the equipment.

3. All participants explicitly stated that they agreed to take part.

The British Psychological Society requirement that all participants give signed consent was one of the most difficult issues to resolve: if all participants were to sign a separate consent form, then a forest of trees would suffer. Suggestions that were made to address this were:

having all the consent signatures on one page: however, this would compromise participants' anonymity;

asking all students to sign a blanket consent at the start of the year that they agree to take part in any other students' evaluations: however, this does not conform completely with the ethical requirements which are that explicit consent needs to be provided;

- 'signed' consent being given by participants though an on-line form, rather than on paper: however, this requires investment in additional programming and administration, and may not stand up to auditing.

A significant concession was made by the Faculty Ethics Committee in agreeing that verbal consent would be sufficient, on the condition that the data was not to be published, nor the results used beyond the term of the project - otherwise, signed consent on separate forms was necessary. We assumed that this latter case would typically arise when the student's project is part of a larger research project for which the supervising academic would have had to apply previously to the Faculty Ethics Committee for ethical approval.

To ensure that verbal consent is sought, the example introduction script includes the explicit question: 'Do you agree to take in this evaluation?

4. No incentives were offered to the participants.

This guideline is to ensure that the payment of participants is not used to induce them to risk harm beyond that which they risk without payment in their normal lifestyle. None of the student assessments or projects are funded to allow for such payment, and most students take part in reciprocal evaluations.

5. No information about the evaluation or materials was intentionally withheld from the participants.

Withholding information or misleading participants is unacceptable if participants are likely to object or show unease when debriefed. The example introduction script provided includes an example of a brief statement of the purpose of the experiment ('to investigate the suitability of these web pages').

6. No participant was under the age of $\mathbf{1 6 .}$ Parental consent is required for participants under the age of 16 . This excludes students from using young family members for evaluation without getting Department Ethics Committee approval, and prohibits them from visiting schools, or asking school students visiting the university to evaluate software, as has been done in the past. While this constraint may appear to prevent projects entailing the implementation of software intended for younger users (as it constrains the extent of appropriate evaluation), it does not make it 
impossible. Our procedures are that Department Ethics Committee approval should be sought in cases like this, and the Department Ethics Committee can advise on appropriate methods for getting parental approval.

7. No participant has an impairment that may limit their understanding or communication. Additional consent is required for participants with impairments. We assume that any student projects involving participants with impairments will be part of a wider academic-led research project that will already have Faculty Ethics Committee approval.

8. I am not in a position of authority or influence over any of the participants.

A position of authority or influence over any participant must not be allowed to pressurise participants to take part in, or remain in, any experiment. While a few of our higher-level students may be tutors for level 1 students, this is rare.

9. All participants were informed that they could withdraw at any time.

All participants have the right to withdraw at any time during the investigation. The statement 'Please remember ...that you are welcome to withdraw at any time' was included in the example introduction script.

10. All participants have been informed of my contact details.

All participants must be able to contact the investigator after the investigation. The statement 'Please take a note of my email address and the email address of my supervisor' has been included in the example debriefing script.

11. The evaluation was discussed with all the participants at the end of the session, and all participants had the opportunity to ask questions.

The investigator must provide the participants with sufficient information in the debriefing to enable them to understand the nature of the investigation. The debriefing script also includes the question 'Do you have any questions about the experiment?'

12. All the data collected from the participants is stored in an anonymous form.

All participant data (hard-copy and soft-copy) should be stored in anonymous form. The only case when this would not be necessary is for a longitudinal study which would carry on after the course of the study: such studies would be covered under the supervisor's application for approval from the Faculty Ethics Committee.

The example introduction script also includes an explanation as to why human participation is necessary ('We cannot tell how good web pages are unless we are those people who are likely to use them), a brief description of the task ('I will give you some time to browse the pages, before asking you some questions'), a brief description of the data collection method ('I will be observing you while you perform the task'), a reassuring caveat ('please remember that is it the system, not you, that is being evaluated'), as well as an opportunity for the participant to ask any questions before the evaluation session starts.

\section{OUR EXPERIENCES}

While we believe that we have come up with the simplest procedure possible that ensures that the guidelines are being followed, there are still several problems with this method.

\subsection{Cultural change}

Although these procedures have been in place since March 2003, and were revised for simplicity in April 2004, considering ethical issues has not become engrained in our culture of supervising, and instances of violation of these procedures are still found one or two of our final year projects. The supervisors need constant reminding - we discovered that it is not sufficient to merely put lightweight procedures into place: as they are so new, both staff and students will need an annual reminder.

\subsection{Penalties for non-conformance}

We cannot make non-conformance a university disciplinary offence (similar to plagiarism), as it is not included in the university's current code of discipline; the only penalty that we can apply is at a departmental level, by a reduction in marks. If students either do not submit the signed form or it is subsequently discovered that the ethical guidelines were not followed, then zero marks can be given for the evaluation: it should be as if the evaluation never happened.

However, while it is ultimately the students' responsibility to ensure that they comply with the 12 points, if the supervisor does not remind the student, or does not discuss the ethics with the student, it does not seem reasonable to penalise the student until this practise is more established in the departmental culture.

More importantly, while this penalty can be used as a threat, it does not change the fact that (known or unknown) unethical practises may take place in the department.

\subsection{Deviation}

Some final year project students deviate slightly from one or more of these points and therefore need to submit an approval request form to the 
Department Ethics Committee. This is sometimes seen as an unwieldy task that may block adventurous projects. In practise, this committee advises the students on completing the form and on appropriate evaluation methods, and such approval is typically easily gained.

\subsection{Complacency}

The 12-point checklist has been put on the reverse side of the plagiarism form that all students submit with all assessments stating that the work is their own - many students sign it as a matter of course, even if their assessment has not involved any human participation.

\subsection{Organisational Learning}

Our experience has been that the use of ethical guidelines not only helps to safeguard the participants in evaluations, it can also play an important role in improving the quality of student work. In those cases when a student has had submit an ethics approval form to the Department Ethics Committee, they have had to document and justify their methodology: this has never been required in the past.

One aspect of student work that we are still unsure about is requirements gathering. Interviewing people and asking them to complete questionnaires are low risk activities, and are by their nature voluntary, so the argument could be made that there is no need to explicitly follow the code. However, requirements gathering could also be seen as a form of evaluation of an existing situation (for example, existing software, or current record keeping procedures), and the information gained about the existing situation may be sensitive. For example, a student on one of our safety-critical systems courses became aware of potential hazards to employees as part of a requirements elicitation exercise that involved their employers and which did not explicitly follow the Glasgow Ethics Code. After consultation with the lecturer, they reported the hazard and ensured that the problem was rectified. The question arises as to the extent to which information may be used outside of a requirements elicitation process that has not explicitly followed the ethical code.

\subsection{Our Assumptions}

When we began to develop the Glasgow Ethics Code, we were aware that it would be necessary to monitor and revise our procedures over time, and the code as presented here is the outcome of several revisions. We continue to monitor the use of this code: in particular, several simplifying assumptions have been made- these will need to be checked regularly:

- That all assessments as part of coursework given at all levels do not require the students to violate the 12 ethical points.

- That student projects conducted as part of an academic's research are covered by that member of staff's Faculty Ethics Committee approval.

- That students read the 12 points before signing the form.

- That supervisors will highlight the need for seeking approval from Department Ethics Committee when any of the 12 points are not complied with within a student evaluation.

\section{CONCLUSION}

It appears that the best way for these procedures to become part of the departmental culture will be by highlighting particular cases: as more and more of the academic and research staff are made aware of ethics issues through specific incidents that relate to themselves, there is more chance that all members of the department will take these ethical issues seriously. Some recent incidents indicate that academic staff have made assumptions that, for example, school students under the ages of 16 can provide data (both within schools or during oncampus school visits), that programs submitted by first year students can be made available to a final year student, and that PDAs are considered 'standard equipment'. Our task is not over: while we may have devised a relatively simple procedure, it is clear that it will need careful monitoring and restating before we can be certain that were are completely fulfilling our ethical duty.

\section{ACKNOWLEDGEMENTS}

Thanks are due to Chris Johnson for his comments on and contribution to this article, and who, together with Phil Gray and Simon Garrod, gave substantial advice and feedback in the preparation of the Glasgow Ethics Code 12-point check list. The Glasgow Ethics Code materials are available from http://www.dcs.gla.ac.uk/ hcp/ethics.

\section{REFERENCES}

British Psychological Society (BPS) Code of Conduct. http://www.bps.org.uk/, 2004. 